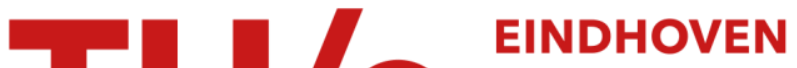 \\ UNIVERSITY OF \\ TECHNOLOGY
}

\section{Adaptive decomposition of noise sources in digital recording systems with media noise}

\section{Citation for published version (APA):}

Beneden, van, S. J. L., Riani, J., \& Bergmans, J. W. M. (2007). Adaptive decomposition of noise sources in digital recording systems with media noise. IEEE Transactions on Magnetics, 43(2), 561-571.

https://doi.org/10.1109/TMAG.2006.887874

DOI:

10.1109/TMAG.2006.887874

Document status and date:

Published: 01/01/2007

\section{Document Version:}

Publisher's PDF, also known as Version of Record (includes final page, issue and volume numbers)

\section{Please check the document version of this publication:}

- A submitted manuscript is the version of the article upon submission and before peer-review. There can be important differences between the submitted version and the official published version of record. People interested in the research are advised to contact the author for the final version of the publication, or visit the $\mathrm{DOI}$ to the publisher's website.

- The final author version and the galley proof are versions of the publication after peer review.

- The final published version features the final layout of the paper including the volume, issue and page numbers.

Link to publication

\section{General rights}

Copyright and moral rights for the publications made accessible in the public portal are retained by the authors and/or other copyright owners and it is a condition of accessing publications that users recognise and abide by the legal requirements associated with these rights.

- Users may download and print one copy of any publication from the public portal for the purpose of private study or research.

- You may not further distribute the material or use it for any profit-making activity or commercial gain

- You may freely distribute the URL identifying the publication in the public portal.

If the publication is distributed under the terms of Article 25fa of the Dutch Copyright Act, indicated by the "Taverne" license above, please follow below link for the End User Agreement:

www.tue.nl/taverne

Take down policy

If you believe that this document breaches copyright please contact us at:

openaccess@tue.nl

providing details and we will investigate your claim. 


\title{
Adaptive Decomposition of Noise Sources in Digital Recording Systems With Media Noise
}

\author{
Steven Van Beneden, Jamal Riani, and Jan W. M. Bergmans \\ Eindhoven University of Technology, 5600 MB Eindhoven, The Netherlands
}

\begin{abstract}
In digital recording systems, the total amount of data-dependent media noise increases considerably as recording densities increase. A proper noise characterization is crucial for the design of receivers for high-density storage systems. This characterization involves the selection of a proper noise model and subsequently the accurate estimation of the parameters of the selected model. The estimation algorithm proposed in this paper jointly estimates the parameters of both media and additive noise with a high accuracy. The proposed algorithm makes use of the data dependency of the media noise to distinguish between the different noise sources. The algorithm is simple and as a result can be implemented in recording systems, with only a limited amount of complexity, as an easy "add-on" to read-channel ICs. From the simulation results and the analytical derivation of the estimation algorithm, we can clearly indicate which data patterns yield near-optimal estimation performance. These patterns are the ideal test patterns in experimental systems. We propose and discuss test patterns for magnetic and optical storage systems.
\end{abstract}

Index Terms-Adaptation, characterization, digital recording, media noise.

\section{INTRODUCTION}

$\mathbf{I}$ $\mathrm{N}$ many recording applications, the noise present in the readback signal can be modeled as a combination of media noise and electronics noise (here referred to as additive noise). Media noise is typically associated with the write process whereas additive noise is associated with the read process [1]. Because the write process is determined by the input data, media noise is strongly data-dependent. It is well known that the presence of data-dependent noise significantly deteriorates the error-rate performance of partial-response maximum-likelihood (PRML) data detection [2]-[5]. Various codes and detectors have been proposed to improve the performance based on specific medianoise models [3], [6], [7]. In an actual system, the performance improvement is only guaranteed if the noise is characterized properly. A proper characterization involves the selection of a model with a proper structure. Furthermore, it involves an accurate estimation of the model parameters based on experimental readback signals. Ideally, the models must be computationally efficient and therefore simple parametric models are commonly used. Parametric models with one and more parameters have been proposed for magnetic systems [8]-[11] and for optical systems [12], [13]. These models are based on the physical nature of the media noise. Hence, the knowledge of the parameter values provides important information for the development and evaluation of recording media as well as detectors for next-generation disk drives. All these parametric models can be considered to be special cases of the signal-dependent autoregressive (AR) channel model [14], [15]. In this model, the media noise is

Digital Object Identifier 10.1109/TMAG.2006.887874

Color versions of one or more of the figures in this paper are available online at http://ieeexplore.ieee.org. the output of an AR system driven by white noise and whose parameters are signal-dependent. The signal-dependent AR model can be transformed by using complex matrix operations into the parametric models that relate well with the physical nature of the media noise [16]. In this paper, parametric models will be used for two reasons: only a few parameters have to be estimated (which results in a limited overall complexity), and the parameter values immediately reveil insightful information about physical properties of the recording channel. In practice, it is highly desirable to be able to characterize the media noise parameters simultaneously with those of the additive noise, i.e., to decompose the noise components of the replay signals into media and additive noise components.

In general, the estimation of parameter values is based on fitting the selected models to readback signals. In experimental systems, one has the freedom to design test patterns and the ability to base the estimation of the parameters on different reads and writes of the test patterns. As a result, a lot of different readback signals are available to base the characterization on, and there is a large flexibility in the design of a characterization procedure. Many existing characterization procedures make use of this flexibility by tailoring the procedure to the specific experimental recording application. For example, to achieve a proper decomposition of the noise sources, the write noise is extracted from the readback signals by appropriately averaging over many reads and writes of a periodic pattern [1].

The topic of this paper is the design of a highly general characterization procedure which is applicable to real-time systems. In real-time and experimental systems, the constraints are very different. The large flexibility available in experimental systems (multiple reads and writes of predefined test patterns) is in great contrast with the single read of a priori unknown patterns available in real-time systems. Hence, in real-time systems much less flexibility is available to design a proper decomposition procedure. Moreover, computational complexity weighs more heavily. In this paper, a decomposition procedure is proposed 
that operates on a single read of an arbitrary pattern and is able to properly decompose the noise sources present in real-time recording systems. The procedure is able to jointly estimate the parameter values of media and additive noise sources by exploiting the data-dependent nature of the media noise. It is computationally simple and very suitable for incorporation as an "add-on" to existing read-channel integrated circuits (ICs).

In any characterization procedure, estimation of the parameter values can be based on spectral or on temporal techniques or on a combination of both [17]. The first class of techniques, often used in experimental environments, involves measuring noise-amplitude spectra for specific test patterns (for magnetic applications, dc and block wave patterns are commonly used) [17], [18]. An advantage of these techniques is the fact that synchronization is not needed. As a result, the analog readback signal can be used as input of a spectrum analyzer and little or no additional signal processing is needed. Furthermore, parameter estimates are obtained by fitting the model to approximate the measured noise spectra. Fitting techniques are often based on the minimization of a suitable criterion or on exhaustive search algorithms, both resulting in a high estimation accuracy [19]. Spectral techniques often rely on highly oversampled readback signals obtained around isolated bit transitions or pairs of transitions [8], [10].

In modern read channels, the analog readback signals are sampled and synchronized to the baud-rate. As a result, it is convenient to base the characterization on the equalized, synchronized, detector-input signal [1], [20]. The second class of estimation techniques (temporal techniques) can use this synchronized signal. In these techniques, the noise correlation matrix is measured based on the synchronized signal and subsequently key portions of this matrix are used to estimate different noise parameters [21], [22]. Temporal techniques are sometimes used in experimental environments where equalized, synchronized signals are available because measuring and fitting the noise correlation matrix are operations with little complexity and still a high estimation accuracy is achieved. In principle, temporal techniques can be applied in real-time environments.

The decomposition procedure proposed in this paper is based on such a temporal technique. More precisely, accurate parameter estimation is achieved based on the open-loop estimation of the ensemble variances of noise samples as a function of the data pattern under consideration. The estimates of the parameter values are obtained by solving a matrix equation with the estimated ensemble variances as input. As opposed to the solution for periodic data patterns presented in [22], it is applicable to arbitrary patterns and therefore it is very well suited for real-time systems. Moreover, our solution is also applicable to experimental systems and we will see that the estimation accuracy is similar to the one achieved by spectral techniques.

The complexity required to calculate the open-loop solution is quite significant and as a consequence it is a disadvantage of the open-loop estimation technique. Hence, a simplification is desired for implementation in real-time environments. A closed-loop version of the procedure provides such a simplification. This closed-loop procedure results in an adaptation algorithm which estimates parameter values on a sample-by-sample basis and requires only a very limited amount of complexity.
Besides this limited complexity, another advantage of the adaptation algorithm is the ability to track variations of media noise parameters. This tracking ability provides information about time-varying artifacts in the recording system and hence gives insight in possible improvements in the recording media and/or read channel.

The adaptation algorithm can be utilized to characterize various types of media noise. In this paper, a model for pit size variations in optical recording systems is used to illustrate the design of the algorithm. In optical recording systems, media noise is due to imperfections in the optical layer of the optical medium, e.g., variations in the dimensions of the pits [23], [24]. This media noise passes through the read-out by the optical spots. Hence, we can say that this media noise originates at the channel input. For example, in CD-RW systems binary input symbols are stored as pits (the laser current is switched on such that a phase change is realized in the recording layer) or lands (no current); only the pits are affected by media noise and not the lands. As a consequence, for such systems media noise can be modeled as noisy pits and noise-free lands. Hence, the media noise is data-dependent. In this paper, we will assume that the media noise samples originate from a white Gaussian stochastic process and that the additive noise samples originate from a correlated Gaussian stochastic process. Exploiting the data-dependency of the media noise, the adaptation algorithm decomposes the different noise sources and provides an estimate of the different parameters, i.e., it performs a joint estimation of the parameters of the media noise and of the additive noise. The analysis and results for this simple optical media noise model can be found in Section II. Because the adaptation algorithm is based on the data-dependency of the media noise and not on any assumption about the media-noise correlation, the algorithm can be extended to cover also correlated media noise.

In Section III, an analysis similar to the one presented in Section II is given for the characterization of media noise in magnetic recording systems. In magnetic recording, position jitter is considered to be the dominant media noise effect [25], yet also other types of media noise arise at high densities, like nonlinear transition shifts and width-variations [26]. All these types of media noise sources are data-dependent and as a result the proposed decomposition procedure can also be applied to magnetic recording channels. A theoretical analysis and simulation results are provided to demonstrate the decomposition capabilities and the estimation accuracy of the proposed adaptation algorithm in magnetic recording applications. Based on the simulation results and the analytical derivation of the adaptation algorithms, one can clearly indicate which data patterns yield near-optimal estimation performance. As a result, these patterns are the ideal test patterns to use in experimental environments. In Section IV, the design of these test patterns for magnetic and optical systems is discussed. Conclusions are provided in Section V.

\section{Media Noise IN OpticAl RECORDING}

A system model of an equalized, synchronized optical recording channel is shown in Fig. 1. The bits $a_{k} \in\{0,1\}$ are inputs of the channel, where $k$ represents the discrete time index, $k=1 \ldots K$ ( $K$ is the total number of transmitted bits), and $a_{k}=0$ for $k \notin[1, K]$. These bits are distorted 


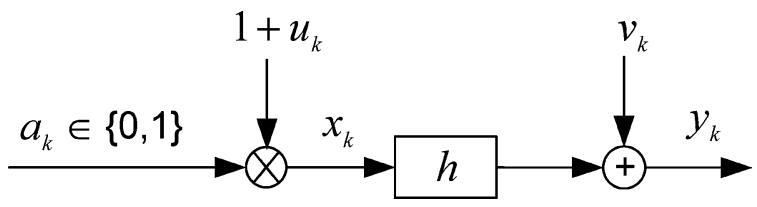

Fig. 1. Model of an equalized, synchronized, optical channel with media noise and additive noise.

by multiplicative noise to produce the channel input signal $x_{k}=a_{k}\left(1+u_{k}\right)$, where $\left\{u_{k}\right\}$ is a sequence of white Gaussian noise samples with zero mean and standard deviation $\sigma_{u}$. As a result, the sequence $\left\{a_{k} u_{k}\right\}$ represents the data-dependent media noise: pits $\left(a_{k}=1\right)$ are noisy while lands $\left(a_{k}=0\right)$ are non-noisy. This is a reasonable first-order approximation of media noise in read-write optical media [24]. In this paper, this specific type of data-dependency is used to illustrate the characterization of two noise sources, but the estimation technique can be applied to other data-dependencies of the noise sources. In the remainder of the paper, the following notation will be used: $\underline{u}=\left\{u_{k}\right\}$ for $k=1, \ldots, K$. The distorted input signal $x_{k}$ is used as input of the channel with impulse response (IR) $\underline{h}=\left[h_{0}, h_{1}, h_{2} \ldots h_{L}\right]$, where $L$ defines the memory span of the channel. Finally a stationary additive noise component $v_{k}$ is present at the output of the channel. This component represents the electronics noise in the recording system and is characterized by the autocorrelation function

$$
R_{v}(n)=\mathrm{E}\left[v_{k-n} v_{k}\right]
$$

for $n=0, \ldots, N$, where $N$ defines the memory length of the additive noise process. Summarizing, the channel output $y_{k}$ can be expressed as

$$
\begin{aligned}
y_{k} & =(h * a)_{k}+(h * a u)_{k}+v_{k} \\
& =\sum_{j} h_{j} a_{k-j}+\sum_{j} h_{j} a_{k-j} u_{k-j}+v_{k}
\end{aligned}
$$

where " $*$ " denotes linear convolution. The first component is the channel output due to the input bits $a_{k}$, and the second component represents the noise at the channel output due to the media noise $a_{k} u_{k}$. The characterization of the noise sources present in this system model concerns the joint estimation of the standard deviation $\sigma_{u}$, or equivalently the variance $\sigma_{u}^{2}$ of $u_{k}$, and the correlation function $R_{v}(n)$ of $v_{k}$ (Section II-A). A key assumption in the estimation is the knowledge of the model output $y_{k}$, the channel IR $\underline{h}$, and the bits $a_{k}$. In a practical system, this knowledge is present at the receiver side: the detector input is readily available, an identification scheme is able to accurately estimate the channel IR. Finally, also the bits are known: either as a specific preamble pattern which used to acquire parameter values in acquisition mode of operation (referred to as data-aided, DA, mode) or as the outputs of a bit detector in tracking mode of operation (referred to as decision-directed, DD, mode). Generally, the bit detector provides fairly accurate bit decisions. However, occasional bit decision errors will affect the overall estimation accuracy. In Section II-C3, we will show that even for a significant bit error rate (BER), up to $10^{-2}$, the estimation accuracy is just slightly influenced.

\section{A. Data-Dependent Media Noise Characterization}

In this section, an algorithm is derived to estimate the parameters $\sigma_{u}^{2}$ and $R_{v}(n)$ based on the observed signal $y_{k}$ and the knowledge of the transmitted data $a_{k}$. To distinguish between the two noise sources $u_{k}$ and $v_{k}$, we have to make use of the data-dependent nature of the media noise. An obvious way to distinguish is to first transmit a long sequence of zero bits followed by random data. In the first part, at the channel output only additive noise is present and as a result $R_{v}(n)$ can easily be estimated. Subsequently, during the random data part the media noise variance $\sigma_{u}^{2}$ can be estimated. Such a procedure is, however, not preferable because the transmission of zero bits decreases the overall information throughput or in some cases it is simply not possible to transmit a sequence of zero bits. For this reason, a procedure that is fully based on arbitrary data patterns is desirable. The procedure developed next applies to arbitrary data patterns. As a result, it is applicable in case random data is present but also short test patterns can be designed to improve the estimation accuracy.

The error signal $e_{k}$ is defined as follows:

$$
e_{k}=y_{k}-(h * a)_{k}=(h * a u)_{k}+v_{k}
$$

for $k=1 \ldots K$. This error signal consists of a data-independent part, i.e., the additive noise $v_{k}$, and a data-dependent part, namely the media noise at the channel output. Based on a datadependent averaging procedure, the data-independent and the data-dependent parts can be characterized jointly. Because the channel IR has a finite memory length $L$, i.e., $h_{k}=0$ for $k \notin[0, L]$, the data-dependency has also a finite memory length. Now define $\underline{a}_{k}^{L}$ as the vector $\left[a_{k}, a_{k-1}, \ldots, a_{k-L}\right]$. The datadependent autocorrelation function of the error signal can be written as

$$
\begin{aligned}
R_{e}\left(n, \underline{a}_{k}^{L}\right) & \triangleq \mathrm{E}\left[e_{k} e_{k-n} \mid \underline{a}_{k}^{L}\right] \\
& =\mathrm{E}\left[v_{k} v_{k-n}\right]+\sum_{j=0}^{L} h_{j} h_{j-n} a_{k-j}^{2} \mathrm{E}\left[u_{k-j}^{2}\right] \\
& =R_{v}(n)+\sigma_{u}^{2} H\left(n, \underline{a}_{k}^{L}\right)
\end{aligned}
$$

where $H\left(n, \underline{a}_{k}^{L}\right)=\sum_{j=0}^{L} h_{j} h_{j-n} a_{k-j}^{2}$. This autocorrelation function can be calculated for $n=0, \ldots, N$ and for all possible data patterns $\underline{a}_{k}^{L}$ which are part of a set $S$. This set $S$ is defined by the modulation code and consists of all permissible data patterns. As a result, a least-squares solution can be formulated for the estimation of $R_{v}(n)$ (for $n=0, \ldots N$ ) and $\sigma_{u}^{2}$. This solution is derived in Appendix A and is given by the solution of the following Yule-Walker equations (in matrix formulation):

$$
\left[\begin{array}{cccc}
\mathcal{H} & H(0) & \cdots & H(N) \\
H(0) & 2^{L} & \cdots & 0 \\
\vdots & \vdots & \ddots & \vdots \\
H(N) & 0 & \cdots & 2^{L}
\end{array}\right]\left[\begin{array}{c}
\sigma_{u}^{2} \\
R_{v}(0) \\
\vdots \\
R_{v}(N)
\end{array}\right]=\left[\begin{array}{c}
\mathcal{G} \\
G(0) \\
\vdots \\
G(N)
\end{array}\right]
$$

where

$$
\begin{aligned}
& H(n)=\sum_{\underline{a}_{k}^{L} \in S} H\left(n, \underline{a}_{k}^{L}\right), \quad \mathcal{H}=\sum_{\underline{a}_{k}^{L} \in S} \sum_{n=0}^{N} H\left(n, \underline{a}_{k}^{L}\right)^{2} \\
& G(n)=\sum_{\underline{a}_{k}^{L} \in S} R_{e}\left(n, \underline{a}_{k}^{L}\right) \text { and } \mathcal{G}=\sum_{\underline{a}_{k}^{L} \in S} \sum_{n=0}^{N} R_{e}\left(n, \underline{a}_{k}^{L}\right) H\left(n, \underline{a}_{k}^{L}\right) .
\end{aligned}
$$


All coefficients $H\left(n, \underline{a}_{k}^{L}\right)$ are only a function of the channel IR $\underline{h}$ and the possible bit patterns (within the memory span of the channel), whereas the coefficients $G\left(n, \underline{a}_{k}^{L}\right)$ depend on the data-dependent autocorrelation function of the error signal.

The accuracy of the estimates is determined by the accuracy of the computed error correlation coefficients $R_{e}\left(n, \underline{a}_{k}^{L}\right)$. As there are $(N+1) \times 2^{L}$ different coefficients for uncoded data, a long averaging period is required to guarantee good estimation performance. The proposed algorithm achieves a one-shot estimation and therefore it is not able to deal with time-varying noise conditions. Moreover, to solve (5), complex matrix operations have to be performed resulting in a computationally intensive procedure. In the next section, a closed-loop adaptive estimation scheme for the noise parameters is proposed which overcomes these disadvantages.

\section{B. Adaptive Estimation Scheme}

The adaptive estimation scheme is based on a stochastic gradient search. The noise parameters are estimated adaptively based on arbitrary data patterns. This approach has the advantage that noise parameter variations can be tracked. The gradient search aims to minimize the cost function defined by (19) (see Appendix B). At every time $k$, a new estimate of the different noise parameters is computed based on the previous estimate and an update term which is the gradient of the cost function with respect to that specific parameter. The adaptation rules are (see Appendix B for the derivation)

$$
\begin{aligned}
{\tilde{\sigma_{u}^{2}}}^{(k)} & ={\tilde{\sigma_{u}^{2}}}^{(k-1)}-2 \mu_{u}\left[\sum_{n=0}^{N} H\left(n, \underline{a}_{k}^{L}\right) \Lambda_{k}(n)\right], \\
\tilde{R}_{v}(n)^{(k)} & =\tilde{R}_{v}(n)^{(k-1)}-2 \mu_{v} \Lambda_{k}(n)
\end{aligned}
$$

for $n=0, \ldots, N$, where $\Lambda_{k}(n)=H\left(n, \underline{a}_{k}^{L}\right){\tilde{\sigma_{u}^{2}}}^{(k-1)}+$ $\tilde{R}_{v}(n)^{(k-1)}-e_{k} e_{k-n}$ is the instantaneous estimation error of the $n$th correlation lag. Here, $\mu_{u}$ and $\mu_{v}$ are the adaptation constants of the media noise update and the update of the additive noise correlation function, respectively. A block diagram of the adaptive estimation scheme is shown in Fig. 2 for $N=0$ (white additive noise). The bits $a_{k}$ and the error signal $e_{k}$ are the only inputs and as they are readily known in read channels, the adaptive estimation scheme can be used as an "add-on" to existing read-channel ICs. The variance estimates ${\tilde{\sigma_{u}^{2}}}^{(k)}$ and $\tilde{R}_{v}(n)^{(k)}$ of the media and the additive noise, respectively, are the outputs. All the coefficients $H\left(n, \underline{a}_{k}^{L}\right)$ for $n=0, \ldots, N$, for all possible $\underline{a}_{k}^{L}$, are computed beforehand and stored in the lookup table (LUT).

1) Steady-State Behavior: The steady-state value of the estimates can be derived easily. For every possible data pattern $\underline{a}_{k}^{L}$, the steady-state values of the estimates can be found by solving the equations obtained by making the expectation of the partial derivatives (20) equal to zero. By applying (4), it can easily be found that $\mathrm{E}\left[\tilde{\sigma}_{u}^{2}\right]=\sigma_{u}^{2}$ and $\mathrm{E}\left[\tilde{R}_{v}(n)\right]=R_{v}(n)$ for all data patterns in $S$.

Furthermore, also the variance of the estimates can be calculated for every data pattern. The derivation is given in Appendix $\mathrm{C}$ for the case the additive noise originates from a white

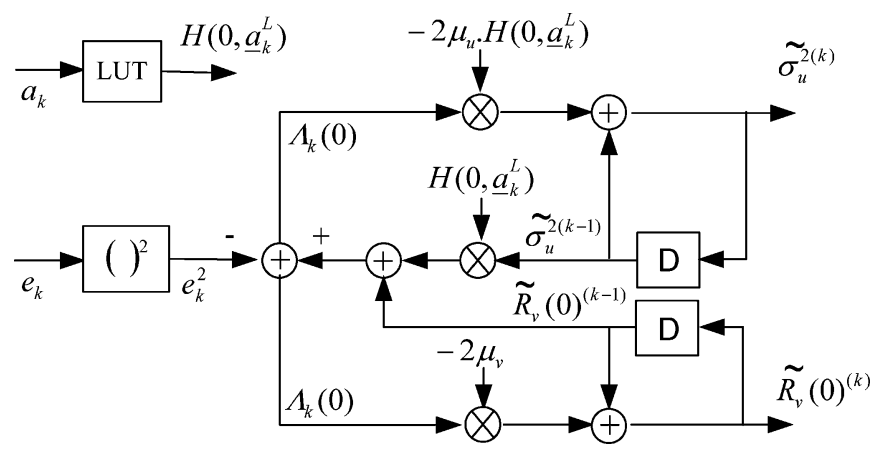

Fig. 2. Model of adaptive noise variance estimation scheme in case media and additive noise originate from independent white AWGN sources. The first input signal $a_{k}$ is used to generate the coefficients $H\left(0, \underline{a}_{k}^{L}\right)$. The second input signal $e_{k}$ together with these coefficients are used to calculate noise variance estimates ${\tilde{\sigma_{u}^{2}}}^{(k)}$ and $\tilde{R}_{v}(0)^{(k)}$.

Gaussian noise source, i.e., $R_{v}(0)=\sigma_{v}^{2}$ and $R_{v}(n)=0$ for $n>0$. The results are

$$
\begin{aligned}
\gamma_{\tilde{\sigma}_{u}^{2}}^{2} & \left.\triangleq \mathrm{E}\left[{\tilde{\sigma_{u}^{2}}}^{2}\right)\right]-\mathrm{E}\left[{\tilde{\sigma_{u}}}^{2}(k)\right]^{2} \\
& \approx 2 \mu_{u}\left(H R_{u}(0)+R_{v}(0)\right)^{2}, \\
\gamma_{\tilde{\sigma}_{v}^{2}}^{2} & \triangleq \mathrm{E}\left[{\tilde{\sigma_{v}^{2}}}^{2}(k)\right]-\mathrm{E}\left[{\tilde{\sigma_{v}^{2}}}_{(k)}\right]^{2} \\
& \approx 2 \mu_{v}\left(H R_{u}(0)+R_{v}(0)\right)^{2} .
\end{aligned}
$$

where $H=H\left(0, \underline{a}_{k}^{L}\right)$ is used to simplify the notation. Based on these expressions, the accuracy of the adaptation scheme can be characterized. It should be noted that these expressions roughly approximate the actual variances for a number of reasons: first by neglecting all higher order terms in (28) and second by the fact that the adaptation scheme is data-dependent (at every time instant a different coefficient $H$ should be used). This data dependency makes the scheme very difficult to analyse accurately. However, in Section II-C we will show that the variances given by (9) are reasonably accurate and can be used as a initial design rule for the choice of the adaptation constants.

2) Example: Assume that the additive noise component $v_{k}$ originates from a white noise process, i.e., $R_{v}(0)=\sigma_{v}^{2}$ and $R_{v}(n)=0$ for $n>0$. In this case, the adaptation rules simplify to

$$
\begin{aligned}
&{\tilde{\sigma_{u}^{2}}}^{(k)}={\tilde{\sigma_{u}^{2}}}^{(k-1)}-2 \mu_{u} H\left(0, \underline{a}_{k}^{L}\right) \\
& {\left[H\left(0, \underline{a}_{k}^{L}\right){\tilde{\sigma_{u}^{2}}}^{(k-1)}+\tilde{R}_{v}(0)^{(k-1)}-e_{k}^{2}\right], } \\
& \tilde{R}_{v}(0)^{(k)}= \tilde{R}_{v}(0)^{(k-1)}-2 \mu_{v} \\
& {\left[H\left(0, \underline{a}_{k}^{L}\right){\tilde{\sigma_{u}^{2}}}^{(k-1)}+\tilde{R}_{v}(0)^{(k-1)}-e_{k}^{2}\right] . }
\end{aligned}
$$

If a sequence of zero bits is transmitted, the media noise variance is not updated and the additive noise variance is changed according to

$$
\tilde{R}_{v}(0)^{(k)}=\tilde{R}_{v}(0)^{(k-1)}-2 \mu_{v}\left(\tilde{R}_{v}(0)^{(k-1)}-e_{k}^{2}\right) .
$$

The latter update rule is the expected one as the error signal $e_{k}$ does not have a component due to the media noise and is only determined by the additive noise. 
Now if random data is transmitted, the coefficient $H\left(0, \underline{a}_{k}^{L}\right)$ is, in general, nonzero. As a result, the update of the media noise variance is enabled and the update represents that part of the error power that is not due to additive noise (addition of $\tilde{R}_{v}(0)^{(k)}$ ) and not yet present in the previous estimate ${\tilde{\sigma_{u}^{2}}}^{(k-1)}$. In the update of the additive noise variance, the term $H\left(0, \underline{a}_{k}^{L}\right){\tilde{\sigma_{u}^{2}}}^{(k)}$ represents that part of the error power $e_{k}^{2}$ that is due to the media noise.

3) Extension to Multiple Media Noise Sources: In most cases, especially at high densities, more media noise sources are simultaneously present in the readback signal. The adaptive decomposition procedure defined in this subsection can easily be extended to the case of multiple data-dependent noise sources. For every parameter, an individual estimation loop has to be used and the data-dependent coefficients of the loop should be tuned to that specific parameter. If the data dependencies of the media noise sources are different, the adaptive decomposition procedure will work and no offsets are present in the variance estimates. The variances of the estimates, given by (9) for the case a single media noise source is present, will contain a term for every noise source (with the corresponding coefficient $H$ ) and as a result the overall variance will increase for increasing number of noise sources.

\section{Simulation Results}

The functionality of the adaptive estimation scheme is illustrated by simulating the model of Fig. 1. An ideally-equalized optical channel is assumed with equalized channel response $h=\left[\begin{array}{lllll}0.17 & 0.5 & 0.67 & 0.5 & 0.17\end{array}\right]$, and channel memory length $L=$ 4. An uncorrelated input sequence $\underline{a}$ is used with $K=4 \times$ $10^{5}$. Furthermore, the media noise sequence $\underline{u}$ is assumed to be white and Gaussian. The signal-to-noise ratio (SNR) is defined as $\mathrm{SNR}=\sum_{j} h_{j}^{2} / \sigma_{z}^{2}$, where $\sigma_{z}^{2}$ is the total noise power: $\sigma_{z}^{2}=\sigma_{u}^{2}+\sigma_{v}^{2}$. We define media noise percentage (MNP) as the ratio of the media noise power to the total noise power [27]

$$
\mathrm{MNP}=\frac{\sigma_{u}^{2}}{\sigma_{z}^{2}}=\frac{\sigma_{u}^{2}}{\sigma_{u}^{2}+\sigma_{v}^{2}} .
$$

In case the additive noise sequence $\underline{v}$ is correlated, the memory length $N$ is in practical situations not known. For this reason, $N$ should be chosen large enough to cover all nonzero elements of the autocorrelation function $R_{v}(n)$. We consider two different cases: $N=0$ and $N=2$.

1) White Gaussian Additive Noise: Here, $R_{v}(n)=0$ for $n>0$ and the memory length $N$ is chosen to be zero $(N=0)$. In Fig. 3, the estimated parameters $\tilde{\sigma_{u}^{2}}$ (upper plot) and $\tilde{\sigma_{v}^{2}}=$ $\tilde{R}_{v}(0)$ (lower plot) are plotted in decibels $(\mathrm{dB})$ versus the sample number for $\sigma_{u}^{2}=0.1$ and $\sigma_{v}^{2}=R_{v}(0)=0.1$ (indicated by the dashed lines in the figure). The adaptation constants are tuned such that the time constant $\tau_{e}$ of both estimation loops are equal and a slow but steady convergence is achieved. To this end, $\tau_{e}=2.10^{4}$ is used. Because on average the media noise variance estimate is updated only half of the time, the adaptation constant $\mu_{u}$ should be a factor of 2 higher than $\mu_{v}$. The different lines in the plot are the results for different noise realizations. Because the control information in the two loops is not completely orthogonal, there is interaction in the estimation
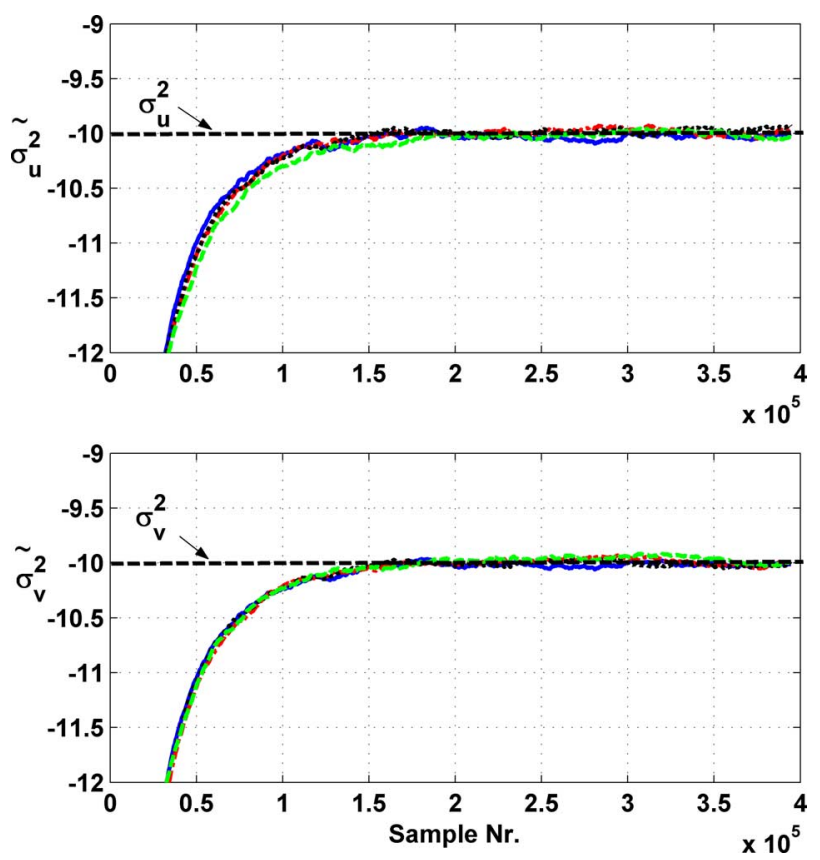

Fig. 3. Estimated parameter values $\tilde{\sigma_{u}^{2}}$ and $\tilde{\sigma_{v}^{2}}$ (given in decibels) versus the sample number. In the simulation, the following values are used: $\sigma_{u}^{2}=0.1$ and $\sigma_{v}^{2}=R_{v}(0)=0.1, \mu_{u}=1 e^{-4}$ and $\mu_{v}=5 e^{-5}$.

of the media noise parameters and the additive noise parameters. This interaction does not introduce a bias in the estimates but influences the convergence to their steady-state values. In steady-state operation, the estimated parameter values do not deteriorate more than $0.02 \mathrm{~dB}$ from their actual values for the given time constant.

In practical situations it will be desirable to have an estimate of the overall SNR and an estimate of the MNP, respectively denoted as $\tilde{\sigma}_{z}^{2}$ and MÑP. These estimates can easily be estimated based on (25) ${\tilde{\sigma_{z}^{2}}}^{(k)}={\tilde{\sigma_{u}^{2}}}^{(k)}+\tilde{R}_{v}(0)^{(k)}$ and $\tilde{M N P}^{(k)}=$ ${\tilde{\sigma_{u}^{2}}}^{(k)} /{\tilde{\sigma_{z}^{2}}}^{(k)}$.

To judge the accuracy of the adaptive estimation scheme, we introduce the normalized estimation accuracy (NEA). The NEA is defined as the deviation in estimated SNR (in $\mathrm{dB}$ ) in case the estimate deviates one standard deviation from its mean value, irrespective of the actual SNR value. More precisely, the NEA can be expressed as

$$
\mathrm{NEA}=10 \log _{10}\left(1+\frac{\gamma_{\tilde{\sigma_{z}^{2}}}}{\sigma_{z}^{2}}\right)
$$

where $\gamma_{\tilde{\sigma}_{z}^{2}}$ is the standard deviation of the SNR estimate. If we assume that the estimates $\tilde{\sigma_{u}^{2}}$ and $\tilde{\sigma_{v}^{2}}$ have independent normal distributions (in reality this is not completely true but it provides a good approximation), this standard deviation can be calculated based on $\gamma_{\tilde{\sigma}_{z}^{2}}^{2}=\gamma_{\tilde{\sigma}_{u}^{2}}^{2}+\gamma_{\tilde{\sigma}_{v}^{2}}^{2}$. The NEA value indicates the accuracy of the estimation with respect to the overall noise power.

In the upper part of Fig. 4, simulated and theoretical NEA values are plotted versus MNP for different values of the adaptation constants $\mu_{v}$ (again $\mu_{u}=2 \mu_{v}$ ) while SNR is fixed at $25 \mathrm{~dB}$. The simulated values are obtained by calculating its expected value over all samples after the estimation loops have converged. The theoretical values are calculated according to 


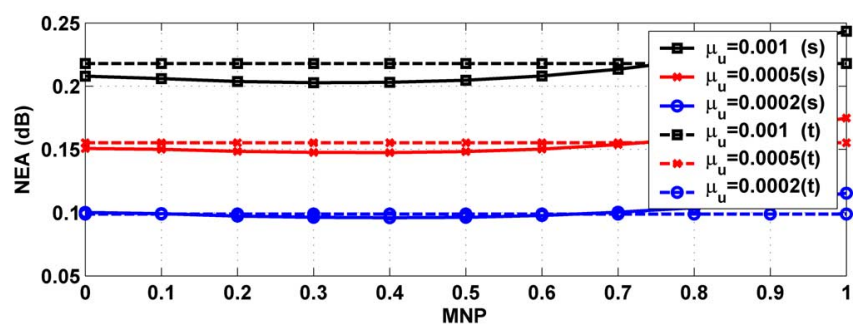

(a)

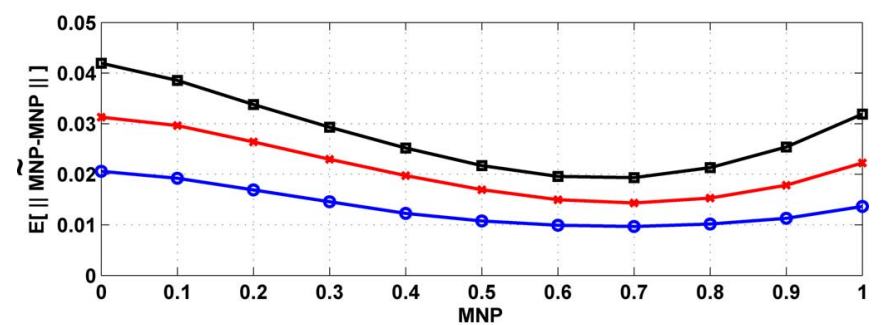

(b)

Fig. 4. (a) Theoretical (t) and simulated (s) NEA values (given in $\mathrm{dB}$ ) versus MNP. (b) Simulated MNP estimation accuracies E[\|MÑP - MNP $\|]$ versus MNP. SNR $=25 \mathrm{~dB}$. Three different adaptation constants are used in the simulations: $\mu_{u}=0.001, \mu_{u}=0.0005$, and $\mu_{u}=0.0002 ;$ and $\mu_{v}=\mu_{u} / 2$.

(13) and (9), where in the latter the maximum value of $H$ is used to account for worst case noise conditions.

From (9), it should be clear that NEA is mainly determined by the square root of the adaptation constant. If the time constant is increased by a factor of 2 , the NEA will decrease by a factor of $\sqrt{2}$ and vice versa. The NEA indicates what is the accuracy of the estimation. For example, the NEA for $\mu_{u}=0.0002$ is approximately 0.1, i.e., the estimated SNR level has a standard deviation of $0.1 \mathrm{~dB}$ from its actual value. This means that you have a probability of $95 \%$ that the estimated SNR is within $0.2 \mathrm{~dB}$ (equal to 2 standard deviations) from its actual value.

In the lower part of Fig. 4, the standard deviation of the estimated MNP is plotted versus MNP for different values of $\mu_{u}$. For example, for $\mu_{u}=0.0002$, this standard deviation is smaller than 0.02 . This means that you have a probability of $95 \%$ that the estimated MNP is within 0.04 of its actual value. From this figure, it should be clear that the standard deviation of the MNP estimate is sensitive to the media noise versus additive noise mix in the readback signal. The best accuracy is achieved in case the magnitude of the noise sources are comparable.

As a conclusion from this plot, it can be stated that sufficient estimation accuracy is achieved in case the time constant $\tau_{e}$ is chosen sufficiently large.

2) Correlated Additive Noise: We consider additive noise with an autocorrelation function $R_{v}(1)=0.47 \times R_{v}(0)$ and $R_{v}(2)=0.19 \times R_{v}(0)$, together with a media noise source. The memory length $N$ is chosen to cover the memory length of the autocorrelation function, i.e., $N=2$. In Fig. 5, the estimated noise parameters (the full lines) are plotted versus the sample number. Also, their actual values are indicated in the figure by the dashed lines. In this example, $\tau_{e}=5.10^{3}$, which induces a lot more gradient noise compared to the case where a higher time constant is chosen (see Fig. 3).

For the given function $R_{v}(n)$ the spectrum of the additive noise has a low-pass characteristic, whereas the channel IR is also low-pass. Because of the data-dependent update, the
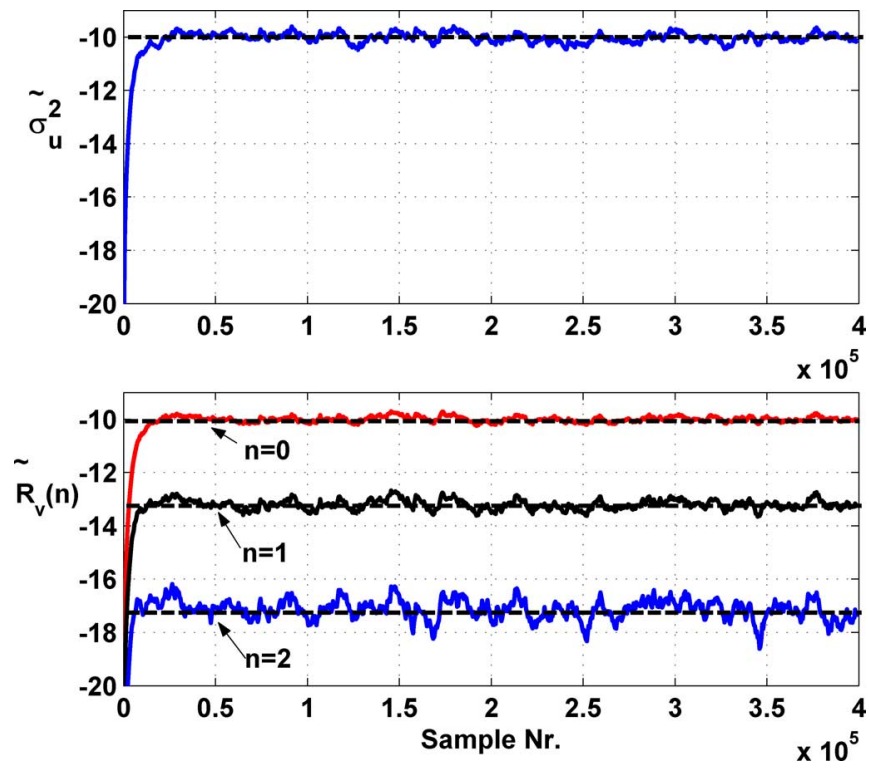

Fig. 5. Estimated parameter values $\tilde{\sigma}_{u}^{2}$ and $\tilde{R}_{v}(n)$ for $(n=0,1,2)$ versus the sample number. In the simulation, the following parameter values are used: $\sigma_{u}^{2}=-10 \mathrm{~dB}, R_{v}(0)=-10 \mathrm{~dB}, R_{v}(1)=-13.2 \mathrm{~dB}$, and $R_{v}(2)=$ $-17.2 \mathrm{~dB}$. In the simulation, the following adaptation constants are used: $\mu_{u}=$ 0.0002 and $\mu_{v}=0.0001$.

two different noise sources can be separated in the estimation process despite the fact they both have a low-pass nature.

As a concluding remark, it can be stated that the noise parameter estimates are fairly accurate if $\tau_{e}$ is chosen sufficiently high, i.e., if the adaptation loops exhibit slow convergence.

3) Decision-Directed Estimation: In the simulation results, we have always assumed that during the estimation we have perfect knowledge of the bit sequence $\underline{a}$. In DD operation mode, however, the bit detector occasionally makes a wrong decision and as a result the accuracy of the estimation algorithm worsens. To assess how the accuracy is influenced, the adaptation procedure depicted in Fig. 1 is simulated. But now instead of taking the actual bit sequence $\underline{a}$ as input, an estimate $\underline{\hat{a}}$ of the bit sequence is used which is produced by a Viterbi detector (VD) based on the detector input signal $y$. The VD is matched to the target response $\underline{h}$ (in case $L=4$ the VD has 16 states). Furthermore, it must be noted that the VD operates optimally in case the white additive noise is present at its input. However, in our case media noise is also present and as a result for MNP values different from 0 , this VD is not optimal in terms of detection reliability. In our simulations, it is used anyway for all MNP values because we are only interested in the effect of bit errors on the estimation accuracy and not in the optimal detection performance for different MNP values.

In Fig. 6, the offsets of the estimated SNR and estimated MNP from their actual values are plotted versus the actual MNP for different BERs. From this figure, it is clear that in case erroneous bit decisions are used as input of the estimation scheme, the estimated SNR will be higher than the actual SNR and the estimated MNP will be lower than the actual MNP. In practical systems, however, the BER can be assumed to be reasonably small $\left(\mathrm{BER}<10^{-2}\right)$ and as a result the estimated SNR and the estimated MNP will exhibit just a small offset from their actual values. These results show that in systems with a BER smaller 

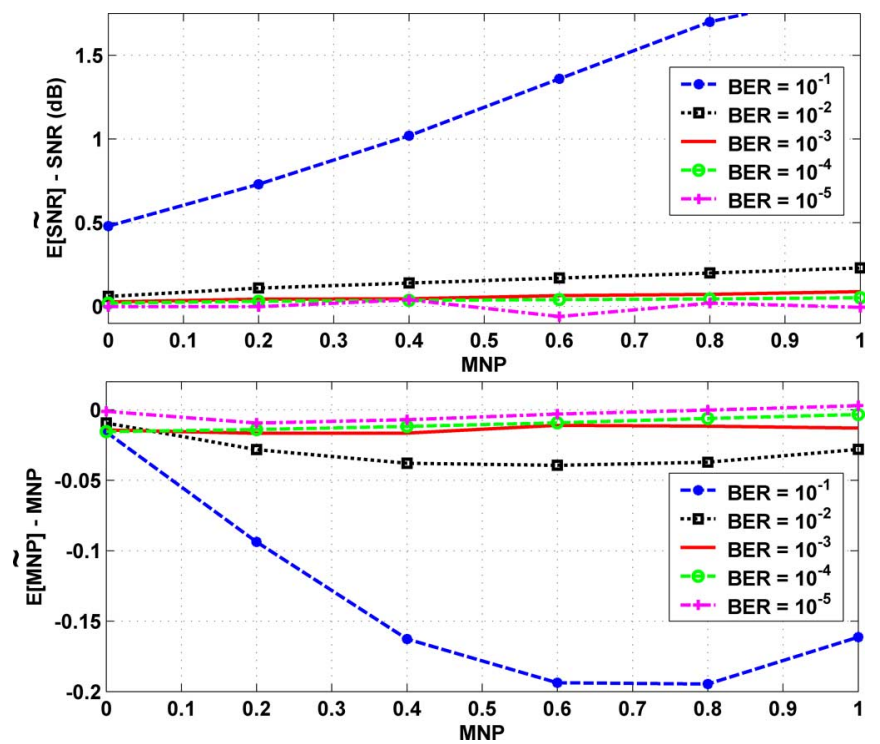

Fig. 6. Offsets of $E[S \tilde{N} R]$ and $E[M \tilde{N P}]$ from their actual values for different BERs.

than $10^{-2}$ the estimation scheme can be used in a DD operation mode.

4) Sensitivity to Channel IR: In the simulation results, we have always assumed that we have perfect knowledge of the channel IR. In practical systems, however, the channel IR is also estimated using an identification scheme. Errors in the estimation of the channel IR will cause a bias in the variance estimate because the coefficients used in the adaptive estimation loop do not correspond to the ones of the actual recording channel. A possible error in the channel IR is a gain mismatch between the actual IR $\underline{h}$ and the estimated IR $\underline{\tilde{h}}$. As we are estimating powers in the adaptive decomposition procedure, gain mismatch can be considered to be the most harmful for a correct operation of the procedure.

In Fig. 7, the offsets of the estimated SNR and estimated MNP from their actual values are plotted versus the actual MNP for different gain factors $\alpha$, where $\tilde{h_{k}}=\alpha h_{k}$ for all $k$. From this figure, it is clear that only relatively large gain mismatches ( $>10 \%$ ) cause a substantial bias in the variance estimates $(\mathrm{E}[\mathrm{MNP}]-\mathrm{MNP}>0.05$ and E[SNR] $-\mathrm{SNR}>1 \mathrm{~dB})$. In practical systems, however, the estimation of coefficients of the channel IR can be assumed to be reasonably accurate if the identification loop is designed well. As a result, in practical systems only a very small bias is to be expected and the channel IR estimation will not affect the adaptive noise decomposition procedure.

\section{MAGNETIC RECORDING}

In magnetic recording applications, position jitter is considered to be the dominant media noise effect [25], yet also other types of media noise arise at high densities, like nonlinear transition shifts and width variations [26]. Furthermore, at the output of the channel, electronics noise is added to the signal. In general, this additive noise is correlated. As a result, in storage systems two independent noise sources are commonly present. Because the media noise is data-dependent and the additive noise is data-independent, they have a different impact on the system

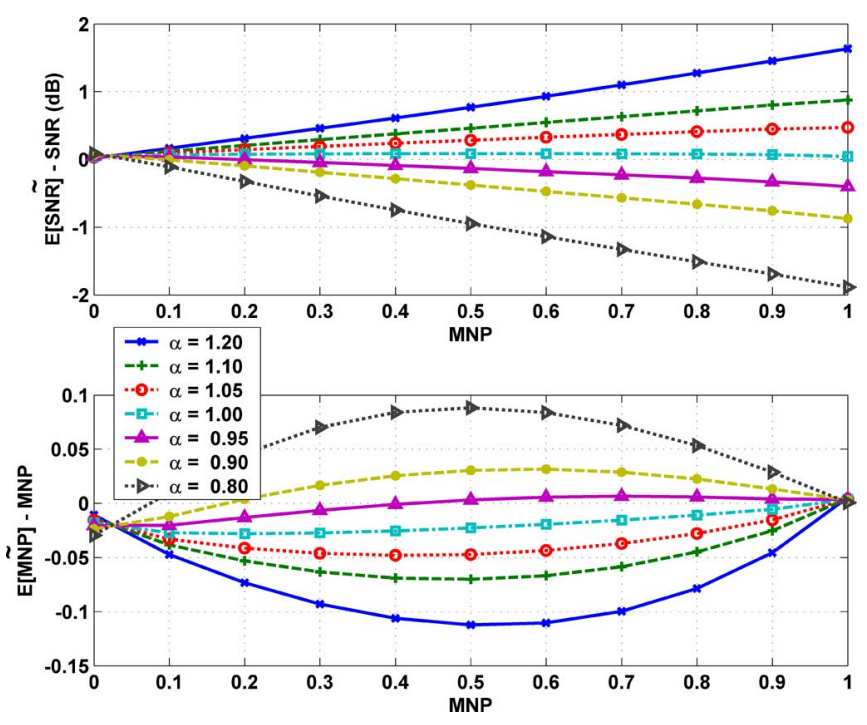

Fig. 7. Offsets of $E[S \tilde{N} R]$ and $E[M \tilde{N P}]$ from their actual values for different gain factors $\alpha$.

and they should be treated separately in a characterization of the storage system. The decomposition procedure used in Section II for optical recording applications, can also be applied to magnetic recording applications. First, in Section III-A, a simplified model for position jitter is proposed on which the rest of this section is based. Subsequently, in Section III-B, an adaptation algorithm for the decomposition of media and additive noise sources in magnetic recording systems is proposed. The proposed algorithm achieves a high estimation accuracy at the expense of limited computational complexity.

\section{A. Media Noise Model}

In this paper, a simplified first-order approximation of position-jitter noise is used as a media noise model. Furthermore, additive noise is also present. The magnetic recording channel model is shown in Fig. 8. The discrete-time output of the model is given by

$$
\begin{aligned}
y_{k} & =\sum_{j} h_{j} x_{k-j}+v_{k} \\
& =\sum_{j} h_{j} d_{k-1-j}+\sum_{j} h_{j}\left(u_{k-j} d_{k-j}-u_{k-2-j} d_{k-2-j}\right)+v_{k}
\end{aligned}
$$

where $\left\{x_{k}\right\}$ is the channel input sequence, $\underline{h}$ is the equalized transition response, $\left\{u_{k}\right\}$ is a random noise sequence, $\left\{d_{k} u_{k}\right\}$ is the media noise sequence reflecting the amount of position jitter, and $\left\{v_{k}\right\}$ is the additive noise sequence. The channel input sequence $x_{k}$ is determined by the transition sequence $d_{k-1}(\epsilon$ $\{-1,0,1\})$ and a random part $u_{k} d_{k}-u_{k-2} d_{k-2}$ due to the transition jitter. This random part is computed somewhat different than in commonly used models [11]. In commonly used models, the effect of transition jitter at the channel output is modeled as the convolution of the media noise sequence with the first derivative of the transition response with respect to time [28]. In our model, this derivative is approximated by the simple $1-D^{2}$ operation which is reasonable because in general $\underline{h}$ is 


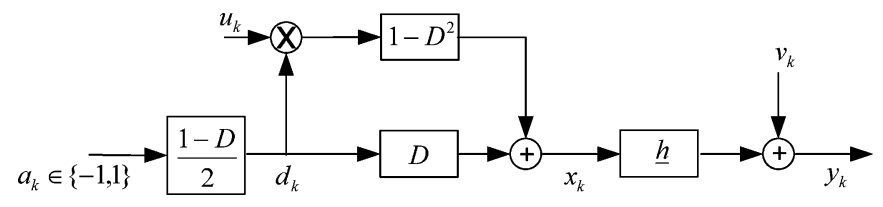

Fig. 8. Simplified magnetic channel model with jitter noise $u_{k}$ and additive noise $v_{k}$.

low-pass and $1-D^{2}$ approximates a differentiator at low frequencies. The transition sequence is obtained from the binary data sequence $a_{k} \in\{-1,1\}\left(d_{k}=\left(a_{k}-a_{k-1}\right) / 2\right)$. All patterns in the sequence $a_{k}$ are part of the set $S$ which is defined by the modulation code.

In this section, the random variables $u_{k}$ and $v_{k}$ are assumed to originate from independent white Gaussian noise sources with variances respectively denoted $\sigma_{u}^{2}$ and $\sigma_{v}^{2}$. The proposed algorithm can be expanded to cover colored noise sources but for notational simplicity we limit ourselves to the white noise case.

The signal-to-noise ratio (SNR) is defined as SNR = $\sum_{j} f_{j}^{2} / \sigma_{z}^{2}$, where $f_{j}=\left(h_{j}-h_{j-1}\right) / 2$ and $\sigma_{z}^{2}$ is the total noise power: $\sigma_{z}^{2}=\sigma_{u}^{2}+\sigma_{v}^{2}$. We define the media noise percentage (NMP) as the ratio of the media noise power to the total noise power [27]

$$
\mathrm{MNP}=\frac{\sigma_{u}^{2}}{\sigma_{z}^{2}}=\frac{\sigma_{u}^{2}}{\sigma_{u}^{2}+\sigma_{v}^{2}} .
$$

From Fig. 8, we can see that that the overall noise consists of two different types of Gaussian noise. One of the types, namely the media noise, depends also on the transmitted signal. Therefore, a solution similar to the one presented in Section II-B can be designed to adaptively decompose the noise components based on the channel output signal.

\section{B. Adaptive Estimation Scheme}

In this section, an algorithm is derived to estimate the stochastic parameters $\sigma_{u}^{2}$ and $\sigma_{v}^{2}$ based on the observed signal $y_{k}$ and the knowledge of the transmitted data $a_{k}$. The algorithm requires a single read of arbitrary data patterns and as a result it can be used in real-time systems. The data-dependent nature of the media noise is used to adaptively decompose the different noise components at the channel output.

An error signal $e_{k}$ is defined as follows:

$$
\begin{aligned}
e_{k} & =y_{k}-\sum_{j} h_{j} d_{k-1-j} \\
& =\sum_{j} h_{j}\left(u_{k-j} d_{k-j}-u_{k-2-j} d_{k-2-j}\right)+v_{k} .
\end{aligned}
$$

This error signal consists of a data-independent part (the additive noise $v_{k}$ ) and a data-dependent part, namely the media noise at the channel output due to the transition jitter sequence $u_{k}$. In most practical data receivers, an equalizer is used to shorten the transition-response memory length to an acceptable duration, such that maximum likelihood sequence detection (MLSD) can be performed with a reasonable complexity [29], [30]. As a result, the equalized transition response $\underline{h}$ can be considered to have a finite memory length $L$, and consequently also the data dependency of the media noise has a finite memory length. Now define $\underline{a}_{k}^{L}$ as the vector $\left[a_{k}, a_{k-1}, \ldots, a_{k-L}\right]$. The power function of the error signal for a specific pattern $\underline{a}_{k}^{L}$ can be written as

$$
\mathrm{E}\left[e_{k}^{2} \mid \underline{a}_{k}^{L}\right]=\sigma_{v}^{2}+\sigma_{u}^{2} H\left(0, \underline{a}_{k}^{L}\right)
$$

where $H\left(0, \underline{a}_{k}^{L}\right)=2 \sum_{j=0}^{L}\left(h_{j}^{2}\left(2-a_{k-j} a_{k-j-1}-a_{k-j-2} a_{k-j-3}\right)\right.$ $+2 h_{j} h_{j+2}\left(a_{k-j-2} a_{k-j-3}-1\right)$. Based on this power function, an adaptive estimation scheme can be derived following the procedure defined in Appendix B. The resulting adaptation rules are the same as obtained in the optical recording example

$$
\begin{aligned}
\tilde{\sigma}_{u}^{2(k)}= & \tilde{\sigma}_{u}^{2(k-1)}-2 \mu_{u} H\left(0, \underline{a}_{k}^{L}\right) \\
& {\left[H\left(0, \underline{a}_{k}^{L}\right) \tilde{\sigma}_{u}^{2(k-1)}+\tilde{\sigma}_{v}^{2(k-1)}-e_{k} e_{k}\right], } \\
\tilde{\sigma}_{v}^{2(k)}= & \tilde{\sigma}_{v}^{2(k-1)}-2 \mu_{v} \\
& {\left[H\left(0, \underline{a}_{k}^{L}\right) \tilde{\sigma}_{u}^{2(k-1)}+\tilde{\sigma}_{v}^{2(k-1)}-e_{k} e_{k}\right] . }
\end{aligned}
$$

The only difference between the optical and magnetic recording example is in the data-dependent coefficients $H\left(0, \underline{a}_{k}^{L}\right)$. More generally for the estimation of different types of media noise the data-dependent coefficients will also be different.

\section{Simulation Results}

The simplified magnetic channel model of Fig. 8 is used to judge the accuracy of the proposed decomposition algorithm. The SNR is set to $20 \mathrm{~dB} ; \sigma_{u}^{2}$ and $\sigma_{v}^{2}$ are varied according to a given MNP. The ME ${ }^{2}$ PRML channel $\left(5+4 D-3 D^{2}-\right.$ $\left.4 D^{3}-2 D^{4}\right)$ is used in the simulations [31], which is shown to be a good match to magnetic recording channels at high densities [32]. The transition response for the $\mathrm{ME}^{2} \mathrm{PRML}$ channel is found to be $\underline{h}=5+4 D+2 D^{2}$. In Fig. 9, the estimated MNP is shown versus the actual MNP for $\mu_{u}=\mu_{v}=0.0001$. The estimated MNP is almost identical to the actual MNP. To judge the achieved accuracy of the adaptation algorithm, the simulated and theoretical NEA values are plotted in the upper part of Fig. 10 for three different time constants $\mu_{u}=\mu_{v}=0.0002$, $\mu_{u}=\mu_{v}=0.0005$, and $\mu_{u}=\mu_{v}=0.001$ and again SNR is set to $25 \mathrm{~dB}$. The theoretical derivation of the estimation variance presented in Appendix $\mathrm{C}$ is also valid for the magnetic recording system and the approximated theoretical curves are also shown. The simulation results are in line with theory. This leads to the same conclusions as in Section II-C, namely that the estimation accuracy is mainly determined by the time constant of the adaptation loops, more precisely by the square root of $\mu_{u}$ and $\mu_{v}$.

In the lower part of Fig. 10, the standard deviation of the estimated MNP is plotted versus MNP for different values of $\mu_{u}$.

\section{Test PATTERn Design}

The adaptive estimation procedure developed here, can be applied to real-time and experimental systems. In the simulation results of Sections II and III, the procedure is applied in a real-time setting, i.e., for a single read of arbitrary, a priori unknown data patterns. In experimental systems, however, one has the freedom to use a sequence of predefined test patterns instead of a single arbitrary pattern. Based on the theoretical analysis of the adaptation algorithm, we will design test patterns for experimental systems (both magnetic and optical recording systems). 


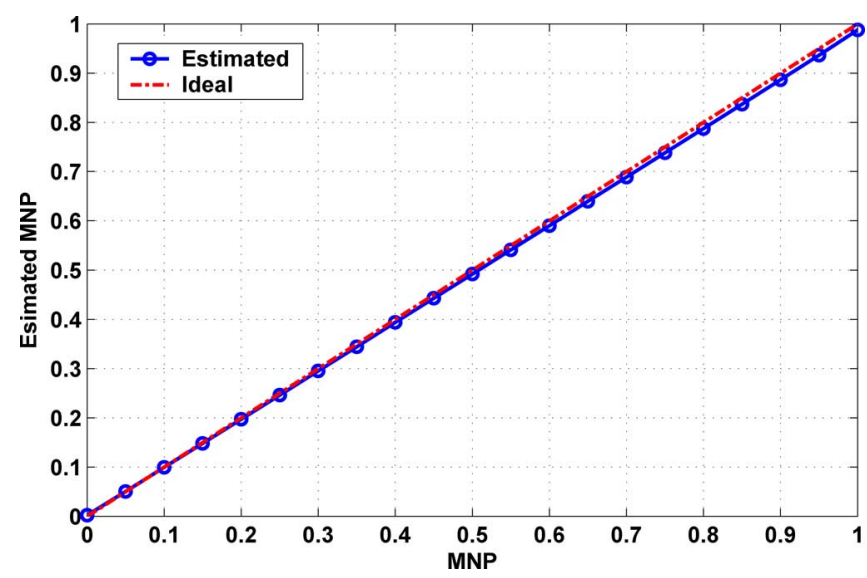

Fig. 9. Estimated MNP: $\mathrm{E}[\mathrm{MNP}]=\mathrm{E}\left[{\hat{\sigma_{u}}}^{2} /{\hat{\sigma_{u}}}^{2}+{\hat{\sigma_{v}}}^{2}\right]$ for $\mu_{u}=\mu_{v}=$ 0.0001 and $\mathrm{SNR}=20 \mathrm{~dB}$.

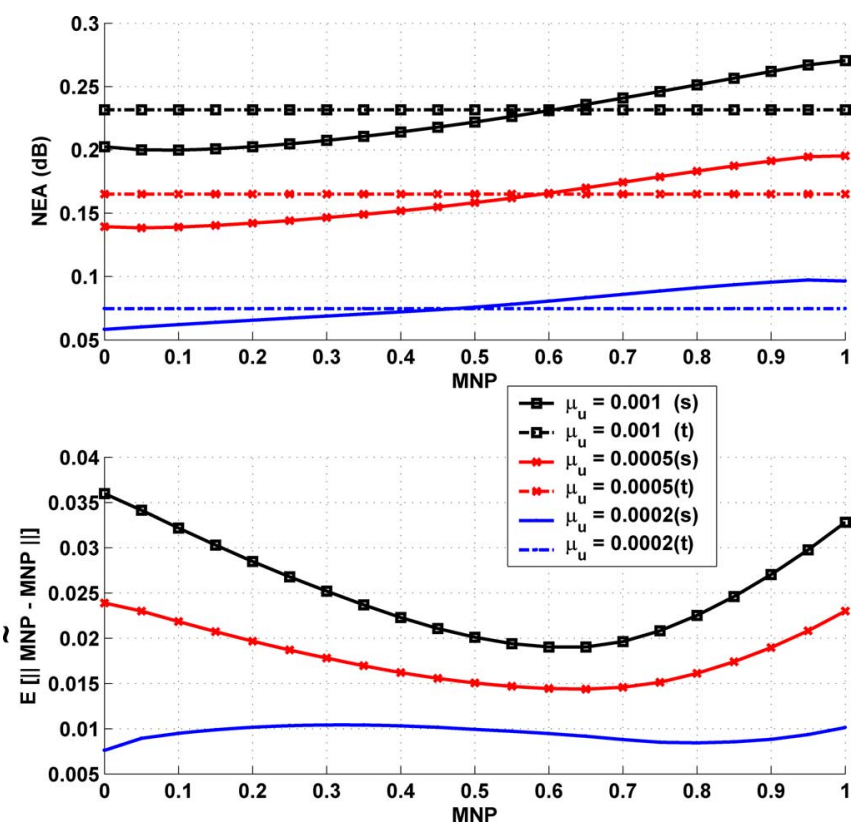

Fig. 10. Simulated (s) and theoretical (t) values of NEA and E[\|MÑ MNP $\|]$ versus MNP for different time constants $\mu_{u}=\mu_{v}=0.0002$, $\mu_{u}=\mu_{v}=0.0005$, and $\mu_{u}=\mu_{v}=0.001$, furthermore SNR $=25 \mathrm{~dB}$.

These test patterns yield an improved estimation accuracy with respect to the accuracy obtained in real-time systems.

The adaptive estimation scheme given by (25) updates the estimated values for every possible data pattern. However, for most of the data patterns a mixture of media noise and additive noise is present and as a result the adaptation of the media noise and of the additive noise interact. This interaction degrades the overall estimation performance. Consequently the algorithm can be changed to update only on data patterns for which one noise source is dominant and as a result also the interaction will be limited.

For the optical channel model presented in Section II, the two opposite data patterns (the all-land pattern 00000 and the all-pit pattern 11111) are of particular interest. The all-land pattern does not induce media noise and therefore the additive noise characteristics should be estimated based on only this pattern. The all-pit pattern, however, induces the maximum amount of media noise information with respect to a fixed amount of additive noise and as a result the media noise estimate should be based only on this all-pit pattern. As a result, the optimal test pattern for optical systems consists of two parts: first an all-land pattern to estimate the additive noise followed by an all-pit pattern to estimate the pit size noise.

In the magnetic channel model presented in Section III, transition jitter is the media noise source under consideration. As transition jitter only occurs if transitions are present in the recorded data sequence, the optimal pattern to estimate the additive noise parameter is the pattern where no transitions occur, namely the all-zero 00000 or the all-one pattern 11111. Consequently, the pattern that should be used to estimate the media noise parameters should have as many transitions as possible, i.e., the Nyquist-pattern 01010. However, in state-of-the-art magnetic recording systems, the channel can be considered to have little or no transfer at the Nyquist frequency and as a result the Nyquist pattern is not very suitable to base the estimation on. Consequently, another pattern with a lot of transitions should be chosen that still has substantial transfer across the channel. A suitable test pattern is the periodic pattern with a period of 2 bits, i.e., 00110011.

Besides the specific test pattern, the accuracy of the estimation algorithm also depends on the knowledge of the channel IR $h$. In practical systems, $h$ is also estimated by an identification scheme. This identification scheme estimates the amplitude of the equalized channel IR. As the estimation of the noise parameters essentially boils down to the estimation of the power of the noise sources, the IR identification scheme is not influenced by the noise estimation. Therefore, a conventional minimum mean square error estimation can be used where the noise in the system results in gradient noise in the estimation of the channel IR. The acquisition of the channel IR in such estimation is guaranteed by the use of a pseudo-noise random sequence. As a result, this sequence needs to be part of the overall test pattern. Furthermore, the adaptive estimation scheme is sensitive to residual even-order nonlinearities (dc-offset, quadratic, $4^{t} h$ order). They have to be carefully compensated for before using the adaptive estimation scheme.

\section{CONCLUSION}

In digital recording systems, the total amount of data-dependent media noise increases considerably as recording densities increase. The estimation algorithm proposed in this paper jointly estimates the parameters of both media and additive noise with a high accuracy. The proposed algorithm makes use of this data dependency to distinguish between the different noise sources. The algorithm is simple and as a result only very limited amount of complexity is required to implement it in recording systems as an easy "add-on" to read-channel ICs. The resulting estimates of the noise parameters provide important diagnostic information about the functionality of the system and possible improvements in the recording system. Simulation results for an idealized optical channel with data-dependent noise show that the estimation of a specific additive or media noise parameter can be very accurate in case its magnitude is not much smaller than the magnitude of the other parameter. 


\section{APPENDIX A}

\section{Derivation of Multivariate Least SQuares Solution}

The multivariate least squares solution is based on the minimization of the total misadjustment power. The misadjustment power is defined as

$$
\begin{aligned}
J & =\sum_{\underline{a}_{k}^{L} \in S} \sum_{n=0}^{N} d\left(n, \underline{a}_{k}^{L}\right)^{2}, \\
& =\sum_{\underline{a}_{k}^{L} \in S} \sum_{n=0}^{N}\left(R_{e}\left(n, \underline{a}_{k}^{L}\right)-\sigma_{u}^{2} H\left(n, \underline{a}_{k}^{L}\right)-R_{v}(n)\right)^{2} .
\end{aligned}
$$

To minimize this cost function, the partial derivatives with respect to the unknown noise parameters are taken and are put to zero

$$
\begin{aligned}
\frac{\partial J}{\partial \sigma_{u}^{2}}= & 2 \sum_{\underline{a}_{k}^{L} \in S} \sum_{n=0}^{N}\left(\sigma_{u}^{2} H\left(n, \underline{a}_{k}^{L}\right)^{2}+R_{v}(n) H\left(n, \underline{a}_{k}^{L}\right)\right. \\
& \left.-R_{e}\left(n, \underline{a}_{k}^{L}\right) H\left(n, \underline{a}_{k}^{L}\right)\right)=0 \\
\frac{\partial J}{\partial R_{v}(0)}= & 2 \sum_{\underline{a}_{k}^{L} \in S}\left(R_{v}(0)+\sigma_{u}^{2} H\left(0, \underline{a}_{k}^{L}\right)-R_{e}\left(0, \underline{a}_{k}^{L}\right)\right)=0 \\
& \vdots \quad \vdots \\
\frac{\partial J}{\partial R_{v}(N)}= & 2 \sum_{\underline{a}_{k}^{L} \in S}\left(R_{v}(N)+\sigma_{u}^{2} H\left(N, \underline{a}_{k}^{L}\right)-R_{e}\left(N, \underline{a}_{k}^{L}\right)\right)=0 .
\end{aligned}
$$

By defining

$$
\begin{aligned}
& H(n)=\sum_{\underline{a}_{k}^{L} \in S} H\left(n, \underline{a}_{k}^{L}\right), \quad \mathcal{H}=\sum_{\underline{a}_{k}^{L} \in S} \sum_{n=0}^{N} H\left(n, \underline{a}_{k}^{L}\right)^{2} \\
& G(n)=\sum_{\underline{a}_{k}^{L} \in S} R_{e}\left(n, \underline{a}_{k}^{L}\right) \text { and } \mathcal{G}=\sum_{\underline{a}_{k}^{L} \in S} \sum_{n=0}^{N} R_{e}\left(n, \underline{a}_{k}^{L}\right) H\left(n, \underline{a}_{k}^{L}\right)
\end{aligned}
$$

the set of equations can be written in matrix form as

$$
\left[\begin{array}{cccc}
\mathcal{H} & H(0) & \cdots & H(N) \\
H(0) & 2^{L} & \cdots & 0 \\
\vdots & \vdots & \ddots & \vdots \\
H(N) & 0 & \cdots & 2^{L}
\end{array}\right]\left[\begin{array}{c}
\sigma_{u}^{2} \\
R_{v}(0) \\
\vdots \\
R_{v}(N)
\end{array}\right]=\left[\begin{array}{c}
\mathcal{G} \\
G(0) \\
\vdots \\
G(N)
\end{array}\right] .
$$

\section{APPENDIX B}

\section{Derivation of AdAPtiVE LEAST MEAN SQuARES SOlution}

The cost function is given by (19). New estimates of the different noise parameters can be computed as

$$
\begin{aligned}
\tilde{\sigma}_{u}^{2(k)}= & \tilde{\sigma}_{u}^{2(k-1)}-\mu_{u} \frac{\partial J}{\partial \sigma_{u}^{2}} \\
\tilde{R}_{v}(0)^{(k)}= & \tilde{R}_{v}(0)^{(k-1)}-\mu_{v} \frac{\partial J}{\partial R_{v}(0)}, \\
& \vdots \quad \vdots \\
\tilde{R}_{v}(N)^{(k)}= & \tilde{R}_{v}(N)^{(k-1)}-\mu_{v} \frac{\partial J}{\partial R_{v}(N)}
\end{aligned}
$$

where $\mu_{u}$ and $\mu_{v}$ are adaptation constants. The partial derivatives with respect to the unknown noise parameters are given by (20). To be able to use these partial derivatives, we replace the expectation values of the error cross correlation by their instantaneous values. Using these partial derivatives in (24) establishes the update rules for the estimation of the noise characteristics:

$$
\begin{gathered}
\tilde{\sigma}_{u}^{2(k)}=\tilde{\sigma}_{u}^{2(k-1)}-2 \mu_{u}\left(\sum_{n=0}^{N} H\left(n, \underline{a}_{k}^{L}\right)\right. \\
\left.\left(H\left(n, \underline{a}_{k}^{L}\right) \tilde{\sigma}_{u}^{2(k-1)}+\tilde{R}_{v}(n)^{(k-1)}-e_{k} e_{k-n}\right)\right) \\
\tilde{R}_{v}(n)^{(k)}=\tilde{R}_{v}(n)^{(k-1)}-2 \mu_{v}\left(H\left(n, \underline{a}_{k}^{L}\right) \tilde{\sigma}_{u}^{2(k)}\right. \\
\left.+\tilde{R}_{v}(n)^{(k-1)}-e_{k} e_{k-n}\right)
\end{gathered}
$$

for $n=0, \ldots, N$.

\section{APPENDIX C STEADY-STATE BEHAVIOR ADAPTATION LOOPS}

The steady-state behavior of the adaptation loops are presented in Fig. 2. Here, the media and the noise additive noise are modeled as AWGN processes. The steady-state value of the estimates can be derived easily. For every possible data pattern $\underline{a}_{k}^{L}$, the steady-state values of the estimates can be found by solving the equations obtained by making the expectation of the partial derivatives given by (20) equal to zero. By applying (4), it can easily be found that $E\left[\tilde{\sigma}_{u}^{2}\right]=\sigma_{u}^{2}$ and $\tilde{R}_{v}(0)=R_{v}(0)$ for all data patterns in $S$.

Furthermore, also the variances of the estimated values $\tilde{\sigma}_{u}^{2}$ can be calculated. Based on the adaptation rules (25), the variances are given by the following expressions:

$$
\begin{aligned}
& \gamma_{{\tilde{\sigma_{u}^{2}}}^{2}}^{2} \triangleq \mathrm{E}\left[{\tilde{\sigma_{u(k)}^{2}}}^{2}\right]-\mathrm{E}\left[{\tilde{\sigma_{u(k)}^{2}}}^{2}\right. \\
& =\left(1-4 \mu_{u} H^{2}+4 \mu_{u}^{2} H^{4}\right) \mathrm{E}\left[{\tilde{\sigma_{u}^{2}(k-1)}}^{2}\right] \\
& +4 \mu_{u}^{2} H^{2} \mathrm{E}\left[{\tilde{\sigma_{v}^{2}}}_{(k-1)}^{2}\right]+4 \mu_{u}^{2} H^{2} \mathrm{E}\left[e_{(k)}^{4}\right] \\
& +\left(8 \mu_{u}^{2} H^{3}-4 \mu_{u} H\right) \mathrm{E}\left[\tilde{\sigma}_{u(k-1)}^{2} \tilde{\sigma}_{v(k-1)}^{2}\right] \\
& -\left(8 \mu_{u}^{2} H^{3}-4 \mu_{u} H\right) \mathrm{E}\left[\tilde{\sigma}_{u(k-1)}^{2} e_{(k)}^{2}\right] \\
& -8 \mu_{u}^{2} H^{2} \mathrm{E}\left[{\tilde{\sigma_{v}^{2}}}_{(k-1)} e_{(k)}^{2}\right]-\mathrm{E}\left[\tilde{\sigma}_{u(k)}^{2}\right]^{2}, \\
& \gamma_{{\tilde{\sigma_{v}^{2}}}^{2}}^{2} \triangleq \mathrm{E}\left[{\tilde{\sigma_{v}}}^{2}(k)\right]-\mathrm{E}\left[{\tilde{\sigma_{v(k)}^{2}}}^{2}\right. \\
& =\left(1-4 \mu_{v}+4 \mu_{v}^{2}\right) \mathrm{E}\left[{\tilde{\sigma_{v}^{2}}}^{2}(k-1)\right] \\
& +4 \mu_{v}^{2} H^{2} \mathrm{E}\left[{\tilde{\sigma_{u(k-1)}^{2}}}^{2}\right]+4 \mu_{v}^{2} \mathrm{E}\left[e_{(k)}^{4}\right] \\
& +\left(8 \mu_{v}^{2} H-4 \mu_{v} H\right) \mathrm{E}\left[\tilde{\sigma}_{u(k-1)}^{2}{\tilde{\sigma_{v}^{2}}}_{(k-1)}\right] \\
& -\left(8 \mu_{v}^{2}-4 \mu_{v}\right) \mathrm{E}\left[\tilde{\sigma}_{v(k-1)}^{2} e_{(k)}^{2}\right] \\
& -8 \mu_{v}^{2} H \mathrm{E}\left[\tilde{\sigma}_{u(k-1)}^{2} e_{(k)}^{2}\right]-\mathrm{E}\left[\tilde{\sigma}_{v(k)}^{2}\right]^{2}
\end{aligned}
$$


where $H=H\left(0, \underline{a}_{k}^{L}\right)$. To simplify these expressions, the expectation values of different signals need to calculated:

$$
\begin{aligned}
\mathrm{E}\left[{\tilde{\sigma_{u(k-1)}^{2}}}^{2}\right] & =\gamma_{\tilde{\sigma}_{u}^{2}}^{2}+\mathrm{E}\left[\tilde{\sigma}_{u(k)}^{2}\right]^{2}, \\
\mathrm{E}\left[{\tilde{\sigma_{v(k-1)}^{2}}}^{2}\right] & =\gamma_{\tilde{\sigma}_{v}^{2}}^{2}+\mathrm{E}\left[\tilde{\sigma}_{v(k)}^{2}\right]^{2}, \\
\mathrm{E}\left[{\tilde{\sigma_{u(k-1)}^{2}}}_{\tilde{\sigma}_{v(k-1)}^{2}}^{2}=\right. & R_{u}(0) R_{v}(0), \\
\mathrm{E}\left[\tilde{\sigma}_{u(k-1)}^{2} e_{(k)}^{2}\right]= & R_{u}(0) \mathrm{E}\left[e_{(k)}^{2}\right], \\
= & H R_{u}(0)^{2}+R_{u}(0) R_{v}(0), \\
\mathrm{E}\left[{\tilde{\sigma_{v}}}_{(k-1)}^{2} e_{(k)}^{2}\right]= & R_{v}(0) \mathrm{E}\left[e_{(k)}^{2}\right] \\
= & H R_{u}(0) R_{v}(0)+R_{v}(0)^{2}, \\
\mathrm{E}\left[e_{(k)}^{4}\right]= & 3 \mathrm{E}\left[e_{(k)}^{2}\right]^{2}=3 H^{2} R_{u}(0)^{2} \\
& +6 H R_{u}(0) R_{v}(0)+3 R_{v}(0)^{2} .
\end{aligned}
$$

The latter equation is derived by applying (4) and the formula of Isserlis [33]. By applying (27), the expressions given by (26) can be simplified:

$$
\begin{aligned}
& \gamma_{{\tilde{\sigma_{u}^{2}}}^{2}}^{2}=\frac{1}{1-\mu_{u} H^{2}}\left[2 \mu_{u} H^{2} R_{u}(0)^{2}+4 \mu_{u} H R_{u}(0) R_{v}(0)\right. \\
& \left.+2 \mu_{u} R_{v}(0)^{2}+\mu_{u}{\gamma_{\tilde{\sigma}_{v}^{2}}^{2}}^{2}\right] \text {, } \\
& \gamma_{{\tilde{\sigma_{v}^{2}}}^{2}}^{2}=\frac{1}{1-\mu_{v}}\left[2 \mu_{v} R_{v}(0)^{2}+4 \mu_{v} H R_{u}(0) R_{v}(0)\right. \\
& \left.+2 \mu_{v} H^{2} R_{u}(0)^{2}+\mu_{v} H^{2} \gamma_{\tilde{\sigma}_{u}^{2}}{ }^{2}\right] \text {. }
\end{aligned}
$$

In normal conditions, the adaptation constants are chosen such that $1>\sigma_{u}^{2} \gg \mu_{u}>0$ and $1>R_{v}(0) \gg \mu_{v}>0$. Based on these considerations, the expressions can be approximated by

$$
\begin{aligned}
& \gamma_{{\tilde{\sigma_{u}^{2}}}^{2}}^{2} \approx 2 \mu_{u}\left(H R_{u}(0)+R_{v}(0)\right)^{2}, \\
& {\gamma_{\tilde{\sigma}_{v}^{2}}{ }^{2}}^{2} \approx 2 \mu_{v}\left(H R_{u}(0)+R_{v}(0)\right)^{2} .
\end{aligned}
$$

\section{ACKNOWLEDGMENT}

This work was supported by the European Union under the “TwoDOS” IST Project IST- 2001-34168.

\section{REFERENCES}

[1] R. D. DeGroat, M. Vis, and W. G. Bliss, "Experimental characterization of media noise based on equalized, sybchronized drive data," IEEE Trans. Magn., vol. 37, no. 2, pp. 633-638, Mar. 2001.

[2] X. Zhang, T. M. Duman, and E. M. Kurtas, "Information rates of binaryinput intersymbol interference channels with signal-dependent media noise," IEEE Trans. Magn., vol. 39, no. 1, pp. 599-607, Jan. 2003.

[3] R. Wood, "Detection and capacity limits in magnetic media noise," IEEE Trans. Magn., vol. 34, no. 4, pp. 1848-1850, Jul. 1998.

[4] J. Fitzpatrick, H. N. Bertram, and X. Che, "The relationship of medium noise to system error rate in a PRML channel," IEEE Trans. Magn., vol. 30, no. 6, pp. 3990-3995, Nov. 1994.

[5] T. R. Oenning and J. Moon, "The effect of jitter noise on binary input intersymbol interference channel capacity," in ICC, 2001 IEEE Int. Conf. Communications, Jun. 11-14, 2001, vol. 8, pp. 2416-2420.

[6] W.Zeng and J. Moon, "Modified Viterbi algorithm for a jitter-dominant $1-D^{2}$ channel," IEEE Trans. Magn., vol. 28, no. 5, pp. 2895-2897, Sep. 1992

[7] A. Kavcic and J. M. F. Moura, "The Viterbi algorithm and Markov noise memory," IEEE Trans. Magn., vol. 46, no. 1, pp. 291-301, Jan. 2000.

[8] Z. Jin, K. Zhang, G. H. Lin, and H. N. Bertram, "Experimental study of off-track dependence of medium noise using a mode projection method," IEEE Trans. Magn., vol. 36, no. 5, pp. 2154-2156, Sep. 2000.

[9] X. Xing and H. N. Bertram, "Error rate analysis of partial response channels in the presence of texture noise," IEEE Trans. Magn., vol. 35, no. 3, pp. 2070-2079, May 1999.
[10] L. C. Barbosa, "A model for magnetic recording channels with signal dependent noise," IEEE Trans. Magn., vol. 31, no. 2, pp. 1062-1064, Mar. 1995.

[11] J. Moon, "Discrete-time modeling of transition-noise-dominant channels and study of detection performance," IEEE Trans. Magn., vol. 27, no. 6, pp. 4573-4578, Nov. 1991.

[12] K. Cai, G. Mathew, J. Bergmans, and Z. Qin, "A generalized BraatHopkins model for optical recording channels," in ICCE, 2003 IEEE Int. Conf. Consumer Electronics, Jun. 17-19, 2003, pp. 324-325.

[13] J. W. M. Bergmans, Digital Baseband Transmission and Recording. Norwell, MA: Kluwer, 1996.

[14] A. Kavcic and M. Srinivasan, "The minimum description length principle for modeling recording channels," IEEE J. Sel. Areas Commun., vol. 19 , no. 4, pp. 719-729, Apr. 2001

[15] A. Kavcic and A. Patapoutian, "A signal-dependent autoregressive channel model,” IEEE Trans. Magn., vol. 35, no. 5, pp. 2316-2318, Sep. 1999.

[16] A. Kavcic and J. M. F. Moura, "Correlation structures for optimizing information criteria," in Proc. IEEE Inform. Theory Workshop on Detection, Estimation, Classification and Imaging, Santa Fe, NM, Feb. 1999.

[17] C. fu, Z. Jin, H. N. Bertram, Y. Wu, and D. Guarisco, "Measurement and analysis of transition noise in perpendicular media," IEEE Trans. Magn., vol. 39, no. 5, pp. 2606-2608, Sep. 2003.

[18] W. C. Williams, B. I. FinkelStein, and T. W. McDaniel, "Spot and mark-size characterization in magneto-optic recording," IEEE Trans. Magn., vol. 24, no. 6, pp. 2323-2325, Nov. 1988.

[19] X. Xing and H. N. Bertram, "Analysis of transition noise in thin film media," IEEE Trans. Magn., vol. 33, no. 5, pp. 2959-2961, Sep. 1997.

[20] W. Zeng, A. Kavcic, and R. Motwani, "Extraction of timing error parameters from readback waveforms," IEEE Trans. Magn., vol. 42, no. 2, pp. 194-199, Feb. 2006

[21] H. N. Bertram and X. Che, "General analysis of noise in recorded transitions in thin film recording media," IEEE Trans. Magn., vol. 29, no. 1, pp. 201-208, Jan. 1993.

[22] H. Pozidis, "Decomposition of noise sources in recording applications using symbol-rate readback samples," IEEE Trans. Magn., vol. 40, no. 4, pp. 2320-2322, Jul. 2004.

[23] A. Stek, G. W. de Jong, T. P. H. G. Jansen, J. R. M. Bergervoet, and P. $\mathrm{H}$. Woerlee, "Circuit design and noise considerations for future blu-ray disc optical storage technology," in Dig. Tech. Papers. ISSCC, Feb. 15-19, 2004, vol. 1, pp. 136-137.

[24] A. Immink, "System and receiver design for two-dimensional optical storage," Ph.D. dissertation, Eindhoven Univ. Technol., Eindhoven, The Netherlands, 2005.

[25] N. M. Zayed, J. C. Park, and L. R. Carley, "Detection for signal-dependent correlated noise in magnetic recording," in ICC '99, IEEE Int. Conf. Communications, Jun. 6-10, 1999, vol. 3, pp. 1972-1976.

[26] A. Taratorin, D. Cheng, and E. Marinero, "Media noise, nonlinear distortions, and thermal stability in high density recording," IEEE Trans. Magn., vol. 36, no. 1, pp. 80-85, Jan. 2000.

[27] I. Ozgunes, B. V. K. V. Kumar, and M. H. Kryder, "Effect of transition noise on the signal-to-noise ratio of magnetooptic read channels," IEEE Trans. Magn., vol. 32, no. 4, pp. 3291-3304, Jul. 1996.

[28] T. Oenning and J. Moon, "Modeling the Lorentzian magnetic recording channel with transition noise," IEEE Trans. Magn., vol. 37, no. 1, pp. 583-591, Jan. 2001.

[29] C. D. Cideciyan, F. Dolivo, R. Hermann, W. Hirt, and W. Schott, "A PRML system for digital magnetic recording," IEEE J. Sel. Areas Commun., vol. 10, no. 1, pp. 38-56, Jan. 1992.

[30] G. D. Forney, "Maximum-likelihood sequence estimation of digital sequences in the presence of intersymbol interference," IEEE Trans. Inf. Theory, vol. IT-18, no. 3, pp. 363-378, May 1972

[31] Z.-N. Wu and J. M. Cioffi, "Low-complexity iterative decoding with decision-aided equalization for magnetic recording channels," IEEE J. Sel. Areas Commun., vol. 19, no. 4, pp. 699-707, Apr. 2001.

[32] H. Sawaguchi, M. Kondou, N. Kobayashi, and S. Mita, "Concatenated error correction coding for high-order PRML channels," in GLOBECOM '98. IEEE Global Telecommunications Conf., Nov. 1998, pp. 2694-2699.

[33] L. Isserlis, "On a formula for the product-moment coefficient of any order of a normal frequence distribution in any number of variables," Biometrica, vol. 12, pp. 134-139, 1918.

Manuscript received August 10, 2006; revised November 7, 2006. Corresponding author: S. Van Beneden (e-mail: s.v.beneden@tue.nl). 